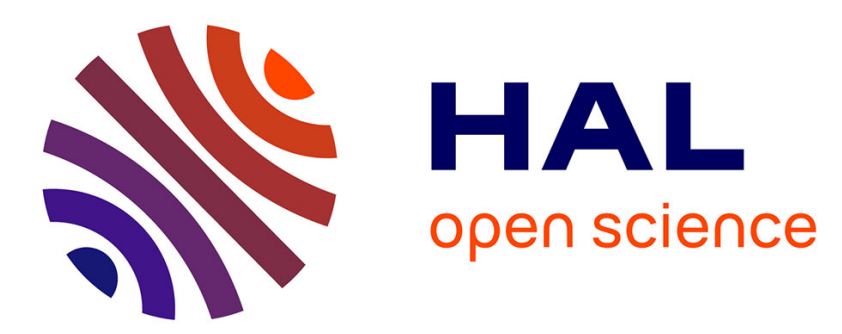

\title{
Modeling breakup and relaxation of Newtonian droplets using the advected phase field approach
} Julien Beaucourt, Thierry Biben, Anne Leyrat, Claude Verdier

\section{To cite this version:}

Julien Beaucourt, Thierry Biben, Anne Leyrat, Claude Verdier. Modeling breakup and relaxation of Newtonian droplets using the advected phase field approach. Physical Review E : Statistical, Nonlinear, and Soft Matter Physics, 2007, 75, pp.021405. 10.1103/PhysRevE.75.021405 . hal-00197263

\section{HAL Id: hal-00197263 \\ https://hal.science/hal-00197263}

Submitted on 14 Dec 2007

HAL is a multi-disciplinary open access archive for the deposit and dissemination of scientific research documents, whether they are published or not. The documents may come from teaching and research institutions in France or abroad, or from public or private research centers.
L'archive ouverte pluridisciplinaire HAL, est destinée au dépôt et à la diffusion de documents scientifiques de niveau recherche, publiés ou non, émanant des établissements d'enseignement et de recherche français ou étrangers, des laboratoires publics ou privés. 


\title{
Modeling breakup and relaxation of Newtonian droplets using the advected phase field approach
}

\author{
J. Beaucourt, T. Biben, A. Leyrat, C. Verdier* \\ Laboratoire de Spectrométrie Physique, Université Joseph Fourier Grenoble I and CNRS (UMR5588), \\ 140 avenue de la physique, BP87 - 38402 Saint Martin d'Hères cedex, France.
}

\begin{abstract}
The relaxation and breakup of Newtonian droplets is considered using the advected field approach. This method allows to follow the deformation of interfaces using an order parameter field [Biben et al., Europhys. Letters, 63(4), 623 (2003)] based on a Ginzburg-Landau equation. Using this method, it is possible to follow the breakup of droplets and stability curves can be obtained both in $2 \mathrm{D}$ and $3 \mathrm{D}$ shear and elongational flows. Finally, relaxation of a droplet is considered, following the application of an elongational flow. The results are compared with previous experimental data [Ha and Leal, Phys. Fluids, 13(6), 1568 (2001)], and are found to be in satisfactory agreement. The method is general enough to be applied to other non-Newtonian fluids, such as Oldroyd-B fluids or viscoplastic materials.
\end{abstract}

\section{Introduction}

The droplet breakup problem was introduced long ago by Taylor $[1,2]$ both experimentally and theoretically. Using roller devices, he was able to produce two dimensional shear and elongational flows and applied them to investigate viscous droplet deformation in a viscous suspending fluid. Such flows can lead to stable droplet deformation or to droplet breakup. In this problem, the capillary number $C a=\frac{\eta_{2} R \dot{\gamma}}{\sigma}$ (where $\eta_{2}$ is the suspending fluid viscosity, $\mathrm{R}$ the radius of the drop, $\dot{\gamma}$ the rate of deformation in shear or elongation, and $\sigma$ is the interfacial tension) and the viscosity ratio $\lambda=\frac{\eta_{1}}{\eta_{2}}$ (where $\eta_{1}$ is the droplet viscosity) are the important dimensionless parameters. Following this approach, Grace [3] demonstrated experimentally that critical deformations and capillary numbers for breakup can be determined as functions of $\lambda$ in shear and elongational flows, over ten decades. This was also investigated in the case of mixed flows [4]. Results from Grace [3] show that it is easier to break droplets in elongation than in shear. A shear flow is indeed the combination of an elongation along the diagonal axes, at 45 from the shear direction, and a rotation tilting the drop. The competition between these two components is controlled by the viscosity ratio, in particular a droplet cannot be broken in a shear flow [3] when the viscosity ratio $\lambda$ is larger than roughly 3.5. At large viscosity ratios, the rotational component of the flow tends to orient the main axis of the drop along the shear direction, where elongational effects are weak.

The shape of the droplets in such flows is also a point of interest. Torza et al. [5] have shown that droplet shapes can vary quite a lot with the viscosity ratio $\lambda$, in particular small ratios lead to droplets with pointed ends. The prediction of droplet deformation has been considered from a theoretical as well as a numerical point of view.

*Author to whom correspondence should be addressed verdier@ujfgrenoble.fr Tel. +33 476635980 Fax +33476 635495
Analytical models based on small deformation theory [6], slender body theory $[7,8]$, or using matched asymptotics [9], have been developed, allowing predictions of the critical capillary number for droplet breakup, also named the onset of "burst". In particular, using perturbations of the flow around the droplet, stability analysis leads to a surprisingly good prediction of the critical capillary number $[6]$, as a function of the viscosity ratio $\lambda$. With the developement of numerical methods such as the boundary integral method [10-14], calculations have also made considerable advances therefore allowing for more intricate shapes to be obtained more accurately. This is of outmost importance when looking at nonlinear effects, as exhibited when stretching filaments or breaking drops into two or more droplets, or forming satellite droplets $[2,15]$.

The numerical technique should not only allow to predict the burst criterion (in a possible large range of viscosity ratios) but should also give the exact details of the droplet rupture sequence, such as the rupture time, the shape of the filament at pinch-off [15-19], the number of droplets and their size [3, 19]. In particular, close attention needs to be paid to the exact significance of the physics involved in the rupture process, in order to determine whether it is initiated by capillary waves [15] or if it has a deterministic nature, related to the well-defined Rayleigh instability [20, 21].

Along with the boundary integral methods, new techniques appeared such as the level-set [19, 22-24], volumeof-fluid method [25], phase field approach [26-31], which allow the computation of two and three-dimensional flows, for various deformable entities under flow, such as droplets or vesicles. The advantage of these new methods relies on their ability to be applied to various constitutive equations describing the inner or outer fluid (Oldroyd-B fluid [24], yield-stress fluid [32]). Also, such problems can be solved in the whole domain, without having to consider explicitly the moving interfaces. The complexity of the deformable object can also be investigated; in particular one may study drops with compatibilizers [33, 34], capsules [35], or vesicles [26]. Finally, the ability of such 
a technique to predict breakup (and coalescence) phenomena in a whole domain seems promising for the understanding of multiphase flows [29, 30], the rheology of emulsions, which have been problems of interest for many years $[1,36-38]$.

In this paper, we propose to test the ability of the advected phase field approach [27] to investigate the dynamics of droplets breakup and relaxation. The main motivation is to compute stability curves as depicted by Grace [3], but also to follow the relaxation of droplets following a step-up in elongation, as studied previously $[12,39,40]$. Numerical simulations will then be compared with previous studies, including experimental results.

In the first part, the model is presented (I). Then finite size effects are presented (II) in the case of the relaxation of a long drop, in the absence of an imposed flow field. Indeed confinement can affect the dynamics of drop relaxation. Stability curves are exhibited and compared with experimental data, as well as previous computations by other authors (III). In the final part (IV), we focus on droplet relaxation, in particular following the paper of $\mathrm{Ha}$ and Leal [40], where extensive data is reported and where the effect of the initial stretch or deformation has been particularly emphasized. Comparisons between numerical data and experiments are discussed.

\section{THE ADVECTED FIELD METHOD (AF)}

The main idea inherent to this method is to consider a fluid-fluid interface as a diffuse locus where a function $\phi$ (phase field) goes smoothly from -1 to +1 . In this case, -1 designates one fluid, and +1 the other fluid component. This method is therefore well adapted for describing binary fluids, in particular immiscible fluids. $\phi$ varies with time $t$ and position $r$ and may be regarded as a rescaled concentration, as in diffusion problems. For the study of such binary systems, one can use a well known freeenergy functional $F[\phi][41]$, a functional of $\phi(r, t)$. An example of such a free energy is the following:

$$
F[\phi]=\int_{V}\left(W(\phi)+\frac{\epsilon^{2}}{2}|\nabla \phi|^{2}\right) d V
$$

where $W(\phi)$ is the following potential

$$
W(\phi)=\frac{1}{4}\left(1-\phi^{2}\right)^{2}
$$

This determines the two equilibrium values \pm 1 , and the shape across a planar interface at equilibrium, which is simply given by $\phi(r)=\tanh (r / \epsilon \sqrt{2})$. Thus the interface thickness is $\epsilon \sqrt{2}$. The temporal evolution equation for the field $\phi$ is given by the advection due to the flow field, and follows an Allen-Cahn approach[42, 43]:

$$
\frac{\partial \phi}{\partial t}+\mathbf{v} \cdot \nabla \phi=-\Gamma\left(\frac{d W}{d \phi}-\epsilon^{2} \Delta \phi\right)
$$

where $\mathbf{v}$ is the velocity field, and $\Gamma$ a constant which is the inverse of a typical relaxation time of the process.
However, this equation is not sufficient for describing a conservative dynamics. It has been used [44] for the description of thermo-capillary flow, but cannot describe the dynamics of mesoscopic droplets. There are two reasons for that. The first one is that we cannot achieve values of $\epsilon$ which are too small (typically less than $10^{-4} R$, $R$ been the droplet size radius). The second is based on the idea that a drop will relax to equilibrium (spherical shape) in the absence of velocity and if the dynamics is conserved, whereas it will vanish if the dynamics is not conserved, this being due to the presence of the interfacial tension $\epsilon \int_{-1}^{+1} \sqrt{2 W(\phi)} d \phi$ [41], implicitly contained in the Allen-Cahn approach, but not coupled to the velocity field, as one would expect. As described earlier $[26,27,45]$, in order to avoid this problem, we propose to add the counter-term, which cancels the effect due to the Allen-Cahn surface tension, noting that the true surface tension $\sigma$ is accounted for in the velocity field dynamics. It is important to note that this counter-term has the advantage to improve in a very efficient way the stability of the small droplets, that would simply disappear in a standard Allen-Cahn formulation. We shall see examples of this fact below. We then resolve:

$$
\frac{\partial \phi}{\partial t}+\mathbf{v} \cdot \nabla \phi=-\Gamma\left\{\frac{d W}{d \phi}-\epsilon^{2}(\Delta \phi+c|\nabla \phi|)\right\}
$$

In this new formulation, $c$ is the local curvature. The reason for this expression is due to an asymptotic expansion, which shows that when $\epsilon$ goes to 0 (sharp interface limit), the main terms cancel out at first order $\epsilon$. The equilibrium shape of the interface is then given again by

$$
\phi(r)=\tanh (r / \epsilon \sqrt{2})
$$

whatever the curvature of the interface, because only the radial component $r$ measured across the area is relevant here. Due to the presence of the restoring term on the r.h.s. of (4), the field $\phi$ now becomes a passive variable whose value is prescribed by the hyperbolic tangent formula. $\phi=0$ corresponds to the real interface locus.

Finally, the velocity field is the solution of the momentum equation:

$$
\rho\left(\frac{\partial \mathbf{v}}{\partial t}+\mathbf{v} \cdot \nabla \mathbf{v}\right)=-\nabla p+\nabla \cdot \boldsymbol{\Sigma}+\mathbf{F}_{i n t}
$$

where $\rho$ is the common density of both fluids, $\mathbf{v}$ the macroscopic velocity field, $\boldsymbol{\Sigma}$ the stress tensor and $\mathbf{F}_{\text {int }}$ is the force due to interfacial tension which is located at the interface and has been added to the momentum balance. In fact, $\mathbf{F}_{\text {int }}$ is simply given by:

$$
\mathbf{F}_{\text {int }}=\frac{c}{2} \sigma \nabla \phi
$$

where $\nabla \phi / 2=\delta(r) \mathbf{n}, \mathbf{n}$ is a unit normal vector to the interface pointed outwards, $\delta(r)$ is a Dirac distribution in the sharp interface limit. Note that the curvature is defined by $c=-\nabla \cdot \mathbf{n}$ (negative for a sphere). The stress 
field $\boldsymbol{\Sigma}$ is given by Newton's law: $\boldsymbol{\Sigma}=2 \eta(\phi) \mathbf{D}$, where $\mathbf{D}$ is the symmetric part of the velocity gradient tensor $\nabla \mathbf{v}$. The viscosity $\eta(\phi)$ is domain-dependent and the system of equations $(4,6,7)$ may be solved together with the incompressibility condition

$$
\nabla \cdot \mathbf{v}=0
$$

The boundary conditions and initial conditions will be specified in what follows. Note that the viscosity is allowed to vary smoothly across the interface by prescribing it to be

$$
\eta(\phi)=\frac{1}{2}\left\{\eta_{1}(1-\phi)+\eta_{2}(1+\phi)\right\}
$$

thus the value corresponding to the location of the interface $(\phi=0)$ is simply the average $\left(\eta_{1}+\eta_{2}\right) / 2$. Note that when using (6), care needs to be taken of the derivative of the viscosity, which is no longer a constant. The method can also allow for density contrasts, but this will not be used here since we are only interested in looking at the influence of the viscosity ratio and capillary number. The other parameter is a Reynolds number $R e=\frac{\rho R^{2} \dot{\gamma}}{\eta_{2}}$, when a shear or elongational rate $\dot{\gamma}$ is applied, and conversely a Suratman number $S u=\frac{\rho R \sigma}{\eta_{2}^{2}}$, when relaxation phenomena are considered, and there is no typical velocity, except the one based on surface tension induced velocity $\frac{\sigma}{\eta_{2}}$. In the case of interest, these two dimensionless parameters (Re and $\mathrm{Su}$ ) are quite small, like in Stokes flow.

The method used is based on the calculation of $\phi$, the pressure $p$, and the velocity field $\mathbf{v}$. $\phi$ is solved for using a finite difference scheme (equation (4)), whereas the velocity field is solved from (6) in the Fourier space. For this purpose, we use a regular rectangular grid, with cubic $(3 \mathrm{D})$ or square elements ( $2 \mathrm{D}$ or $3 \mathrm{D}$-axisymmetric). The spacing is chosen to be exactly $\epsilon$ whose value is taken to be $0.04 R$. The flow field $\mathbf{v}$ can be divided into two components; the undisturbed external flow field $\mathbf{v}_{\text {ext }}$ (shear or elongational flow), plus a contribution $\mathbf{u}$, which is due to the flow induced by the droplet. The boundary conditions have been chosen to be periodic for the advected field $\phi$, and also for $\mathbf{u}=\mathbf{v}-\mathbf{v}_{\text {ext }}$.

This enables to solve for relatively small droplets, but satellite droplets smaller than $\epsilon$ are obviously not visible. In such cases, other more technical methods may be relevant such as the ones used in solidification problems, like the Boundary Element Method (BEM) of Almgren [46] or the phase field model by Wheeler and coauthors [47].

\section{FINITE SIZE EFFECTS}

It is important to analyze the strong influence of finite size effects on the breakup sequence, for example when following the relaxation of a drop in a quiescent fluid. Such effects are now investigated. To discuss this point

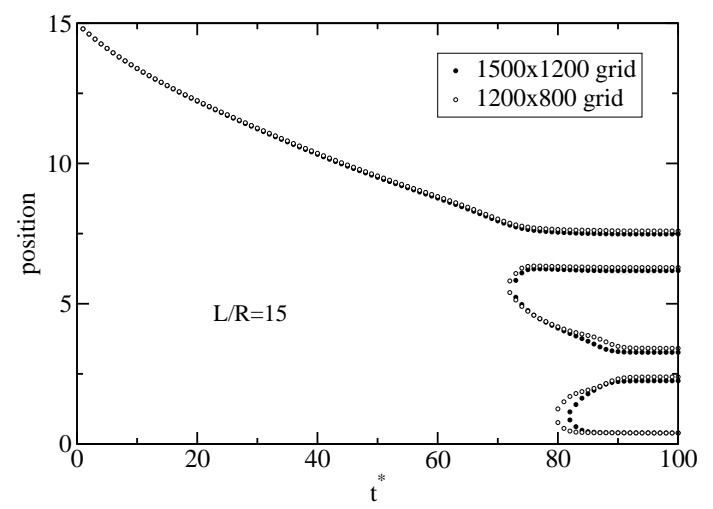

FIG. 1: Fragment edges trajectories for $L / R=15$ and two different box sizes. Similar scenarios

in more details we specify here the geometry we considered and the values of the resolution parameters such as the grid spacing and its size. Since we mostly considered $2 \mathrm{D}$ and $3 \mathrm{D}$-axisymmetric situations, the resolution box is a rectangle of size $N_{x} h \times N_{y} h$, where $h$ is the lattice spacing (here we consider square elements). In 2D, $x$ and $y$ denote the usual coordinates, while in a $3 \mathrm{D}$-axisymmetric geometry, $x$ corresponds to the coordinate along the axis and $y$ the radial coordinate. A droplet is initially placed at the center of the grid. Since we make extensive use of Fourier Transformations, we still consider the full grid for 3D-axisymmetric situations, while a direct space implementation could reduce the problem to a quarter of the grid only, accounting for the symmetries. The boundary conditions on the velocity field are chosen to reduce the boundary effects: we considered periodic boundary conditions for the counter flow induced by the drop, as specified in part I. We fixed $h=0.04 R$ in order to have a sufficient resolution to describe the rapid variation of the Advected Field across the interface, and to have a small enough value of $\epsilon$, the interfacial width introduced by the phase field model. All the results reported in parts III and IV have been obtained with $N_{x}=1200$ and $N_{y}=800$, corresponding to a box size $48 R \times 32 R$ in units of the drop radius at rest. We also considered a smaller box $N_{x}=800$ and $N_{y}=200(32 R \times 8 R)$ that revealed to be too small to provide robust data.

We considered the simple case of a spherocylindrical drop of initial half-length $L / R=15$ ( $L$ half length, $R$ radius of the equivalent spherical drop) to investigate the influence of finite size effects and varied $N_{x}$ and $N_{y}$ in quite a large range: $800 \leq N_{x} \leq 1500$ and $200 \leq N_{y} \leq 1500$. A very sensitive test is to analyze the fragmentation sequence at $L / R=15$ for example. We also tested the breakup sequence for $L / R=18$ and a $1500 \times 1500$ grid. We consider as equivalent two fragmentation sequences for which only small quantitative differences are observed, as shown in Fig.1. 


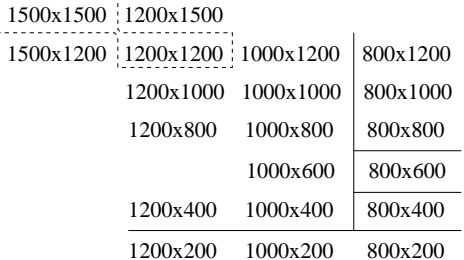

TABLE I: Summary of the data obtained for the relaxation of a spherocylindrical drop with an initial half-length $L / R=15$. The straight solid lines separate regions with very different fragmentation sequences while dashed lines correspond to apparent differences only.

In table I, we summarize the results obtained for $L / R=15$. A full line separating two different box sizes indicates different fragmentation sequences, while no line indicates a similar sequence. The dashed line does in fact correspond to equivalent results, but due to the proximity of a transition between 5 fragments for $L / R=15$ and 7 fragments for $L / R=15.1$ (with a $1200 \times 800$ grid), a slight deviation of the transition point induces an apparent difference in the fragmentation sequence. We consequently also explored neighboring values of $L / R$ to check this feature. From table I, $1200 \times 800$ seems to be a good compromise between accuracy and computational speed. Therefore, in what follows, we will use such a mesh corresponding to a box size $48 R \times 32 R$.

\section{STABILITY CURVES}

We first investigate the ability of the method to predict deformation and breakup of drops accurately. In order to do so, we consider Buckmaster and Flaherty's analytical solution [48] of the flow around a droplet placed in a $2 \mathrm{D}$ elongational flow. The $2 \mathrm{D}$ flow corresponds to $v_{e x t}^{x}=\dot{\gamma} x, v_{e x t}^{y}=-\dot{\gamma} y$, where $x$ is the coordinate along the axis of the drop. The authors [48] were able to calculate the deformation of the drop up to the burst transition. The results of our simulations are presented in Fig.2 and compared with their approximate theory. Below a critical capillary number $C a_{c}$, the drop deforms until it reaches a steady shape [3] that can be characterized by its elongation $1-B / L$, where $B$ is the half-width, and $L$ is the half-length. $B / L$ being always smaller than 1 , the drop has a prolate shape. Above $C a_{c}$, the drop is elongated until it breaks. Such a behavior corresponds to a saddle node bifurcation, thus an unstable branch exists which merges with a stable one, at a critical capillary number. This number is determined by the maximum of the curve in Fig.2, giving approximately $C a_{c} \simeq 0.18$, according to their results. Fig. 2 also shows a good comparison between the theory [48] and results $(\lambda=1)$ obtained with the present AF method. The predictions of the AF method are quite accurate. The analysis of the two branches is as follows.

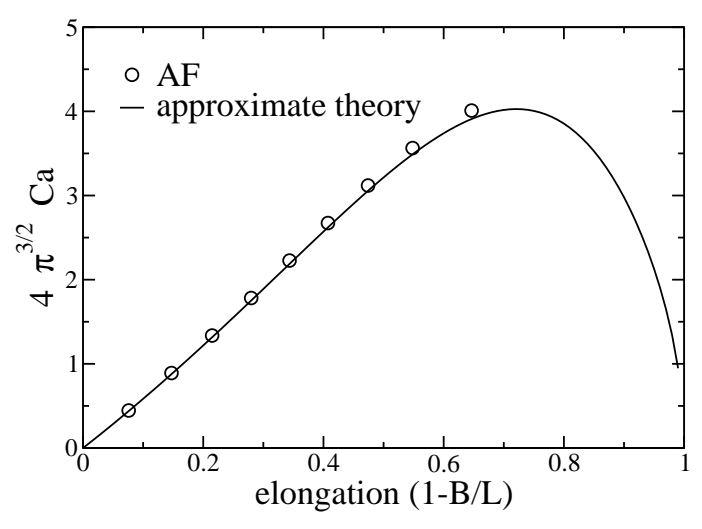

FIG. 2: Capillary number vs. elongation parameter $1-B / L$ $(\lambda=1)[48]$

For a given flow rate, i.e. given capillary number, there are two solutions. The solution corresponding to $1-$ $B / L<0.72$ (stable branch) gives the proper solution, whereas the one corresponding to $1-B / L>0.72$ gives the unstable one. This type of analysis is the starting point for obtaining stability curves at different capillary numbers.

The case of a drop subjected to a shear flow or an elongational flow is considered next. The results are shown in Fig.3. Both 2D and 3D results are also produced. Our analysis is concerned with 2D-shear flows (where $v_{\text {ext }}^{x}=\dot{\gamma} y, v_{\text {ext }}^{y}=0$ ), 2D-elongational flows as shown above, and finally 3D-axisymmetric elongational flows where the flow field is $v_{e x t}^{x}=\dot{\gamma} x, v_{e x t}^{r}=-\dot{\gamma} r / 2$, in cylindrical coordinates, and $x$ is the direction of elongation whereas $r$ is the radial coordinate. This last case is different from the usual four-roll apparatus used in previous studies [4], which assumes a $2 \mathrm{D}$ elongational flow. Critical capillary numbers have been obtained in all these cases, and are compared with results from Grace's work [3] both in shear and in elongation (Fig.3). Also shown is the data from Bentley and Leal [4]. The results obtained with the AF method are in good agreement with the data from the experiments, in particular in the case of elongational flows [3, 4]. While 3D simulations give a very good agreement with the experimental data in this case, we can note that the $2 \mathrm{D}$ data differ significantly. The quantitative difference is even larger in the shearing situation, where the $2 \mathrm{D}$ simulated data can only be compared qualitatively with the 3D experimental geometry. Apart from the quantitative discrepancies, it is interesting to note on the contrary the qualitative agreement between $2 \mathrm{D}$ and $3 \mathrm{D}$ situations. Our study was limited to viscosity ratios varying between 0.01 and 100 . This is already a very satisfactory result, covering four decades in $\lambda$. When small viscosity ratios are considered (say $\lambda<0.01$ ), droplets exhibit pointed ends, and the method 


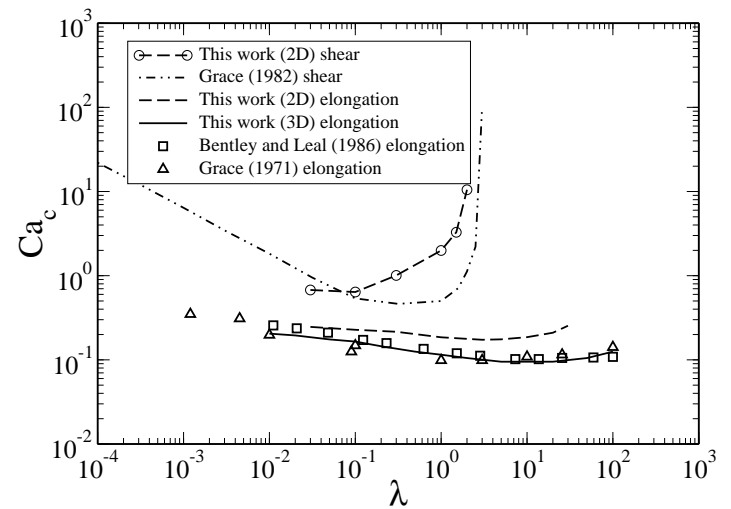

FIG. 3: Critical capillary number $C a_{c}$ vs. viscosity ratio $\lambda$ in $2 \mathrm{D}$ and $3 \mathrm{D}$ shear and elongational flows

fails to predict such shapes because it is unable to account for such large curvatures. Indeed, the small radius of curvature at the ends becomes close to the size of the mesh $\epsilon$, therefore the method cannot give accurate solutions. This AF method is also in agreement with the small deformation theory [6], which predicts well the data in this region, as pointed out by the authors in previous work [27]. Computations in 2D-shear flow are also of interest since they provide evidence of a stable region at viscosity ratios above roughly $\lambda=4$. We observe quantitative differences from the 3D experimental case [3], but we are not aware of such data for the 2D-burst criterion. Our data is not exhaustive, in particular $3 \mathrm{D}$ simulations of shear flow are not provided due to the long times involved for carrying out such computations. Part of this data is available in another paper [25].

\section{DROPLET RELAXATION}

The relaxation of droplets following an elongation is an interesting problem, since it poses the question of what governs the number of fragments, and whether or not the drop relaxes to its initial state. It has been explored both experimentally [39, 40, 49-51] and numerically $[12,50,51]$. It seems well known now that the initial deformation controls the fate of the relaxing droplet, but parameters like the capillary number used to stretch the droplet are also of importance, as well as the viscosity ratio $\lambda$. Recently, Ha and Leal [40] took over the problem of relaxation following a step up in elongation, using their four-roll mill apparatus. At low Reynolds numbers, they showed that the critical stretch ratio increases sharply with the capillary number, when the latter is greater than its critical value for breakup. Also, an interesting feature occurs in the case of stretching followed by relaxation (Newtonian droplets) which is a restabiliza- tion of the droplet following relaxation, when increasing the initial stretch ratio, at a fixed capillary number. The droplet relaxes first to a sphere at small initial stretch ratio, then into two droplets (first transition), then relaxes into a sphere again, and finally into three droplets (second transition). This feature was observed at a viscosity ratio $\lambda=0.056$ and for $C a / C a_{c}=2.15$ [40]. Therefore, more investigations remain to be done in order to see whether this feature is always present, and if such a cascade of restabilizations is possible.

Such an approach is now conducted, based on this interesting set of data [40]. We consider here a 3Daxisymmetric situation.

Before discussing in details the results, we shall first identify the parameters entering in this problem, and the role they play. The elongation-relaxation experiments are essentially controlled by three parameters: The capillary number $C a$ (or alternatively $\mathrm{Ca} / \mathrm{Ca}$ ), the stretch ratio $L / R$, which corresponds to the maximal elongation of the drop, and the viscosity ratio $\lambda$. Whereas these three parameters play a role during the elongation stage, and thus determine the shape of the drop that will suffer the relaxation, only $\lambda$ plays a role in the relaxation stage (for a given initial shape). During this stage, the capillary number is indeed not defined (no applied flow), and the capillary time can be absorbed by a redefinition of the time scales. We shall thus mainly focus on $\mathrm{Ca} / \mathrm{Ca}_{c}$ and $L / R$ to control the initial shape of the relaxing drop, and the particular role of $\lambda$ will be briefly discussed.

\section{A. Comparison with experiments}

We consider in this section the experimental situation of Ha and Leal where the drop is elongated until it reaches a given stretch ratio, and follows a free relaxation afterwards. A first set of data is obtained for $\mathrm{Ca} / \mathrm{Ca} a_{c}=1.05$, and a viscosity ratio $\lambda=0.2$. These values are very similar to those used experimentaly by $\mathrm{Ha}$ and Leal (they used $\lambda=0.209$ and found $C a_{c}=0.135$, whereas here $\lambda=0.2$ and $\left.C a_{c}=0.151\right)$. The results are presented in Fig.4, showing the effect of a change in the stretch ratio $L / R$, where $L$, the half length of the droplet, has been defined previously. This enables to determine a critical stretch ratio $(L / R)_{c}$ above which the drop starts to break during the relaxation stage. Fig. 4 shows the variation of the drop elongation with time. The instantaneous stretch ratio shall be noted $L(t) / R$ and must not be confused with the maximum stretch ratio that we called $L / R$. The features of the curves $L(t) / R$ are as follows: when $L / R$ is smaller than roughly 3.9 , the drop is stretched and relaxes to a spherical shape, as expected, whereas if $L / R$ is larger than 3.9, the drop relaxes into two droplets. Note that a satellite droplet is also observed $(L / R=4.2)$, with a very small size of the order of a few times the value of $\epsilon$. Attention thus needs to be paid regarding this feature both in simulations and in experiments. Our observations are however in good agreement with the ex- 


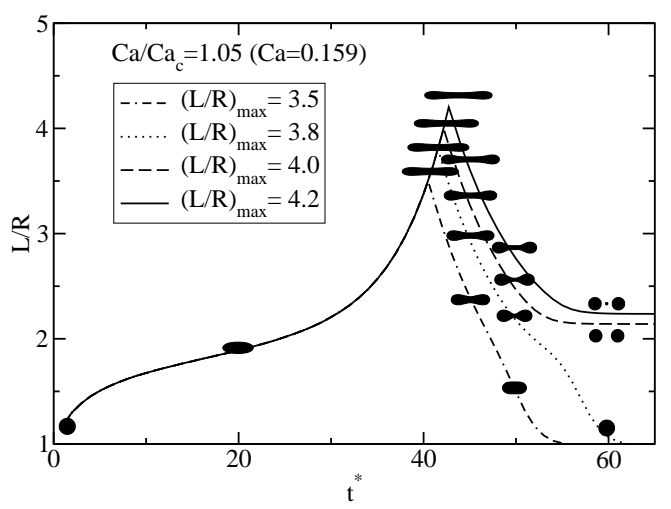

FIG. 4: $C a=0.159 . \quad C a_{c}=0.151 . C a / C a_{c}=1.05$. Relaxation patterns at different values of $L / R(3.5 ; 3.8 ; 4.0 ; 4.2)$ and $\lambda=0.2 \cdot t^{*}=t \sigma / \eta_{2} R$.

\begin{tabular}{|c|c|c|c|}
\hline $\mathrm{L} / \mathrm{R}$ & $\mathrm{N}$ & comment & stable \\
\hline \hline 6.5 & 1 & & $\mathrm{x}$ \\
\hline 7.0 & 1 & & $\mathrm{x}$ \\
\hline 7.1 & 1 & & $\mathrm{x}$ \\
\hline 7.2 & 2 & & $\mathrm{o}$ \\
\hline 7.4 & 2 & & $\mathrm{o}$ \\
\hline 7.45 & 3 & satellite central drop & $\mathrm{o}$ \\
\hline 7.8 & 3 & satellite central drop & $\mathrm{o}$ \\
\hline 7.9 & 2 & no central drop & $\mathrm{o}$ \\
\hline 8.0 & 3 & small central drop & $\mathrm{o}$ \\
\hline 8.4 & 3 & small central drop & $\mathrm{O}$ \\
\hline 8.5 & 5 & alternated large and satellite drops & $\mathrm{O}$ \\
\hline
\end{tabular}

TABLE II: Breakup events for different $L / R(\lambda=0.2$. $C a=$ $\left.0.326, C a / C a_{c}=2.11\right)$. $\mathrm{N}$ is the number of fragments.

periments of Ha and Leal.

While the previous example corresponds to a capillary number very close to the critical capillary number $\mathrm{Ca} / C a_{c}=1.05$, it is interesting to consider larger values. We can carry out a similar analysis for $C a / C a_{c}=2.11$ (and still $\lambda=0.2$ ). The results are presented in Table II and show similarities with the previous example (a succession of one, two and three fragments with a satellite central drop while increasing the stretch ratio $L / R$ ), but more interesting is the restabilization sequence.

The transition from two to three droplets occurs between $L / R=7.4$ and $L / R=7.45$. Then a restabilization is observed for $L / R=7.9$ for which two fragments are produced. Again, we find the formation of three fragments at $L / R=8.0$, indicating that the region of restabilization was very small. Whereas Ha and Leal reported a restabilization scenario from two to one droplet (at a viscosity ratio of $\lambda=0.056$, that we shall consider be-

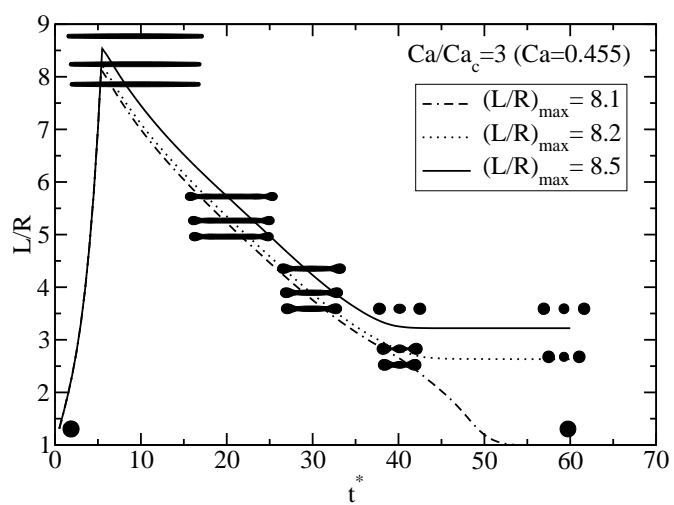

FIG. 5: $C a=0.455 . \quad C a_{c}=0.151 . \quad C a / C a_{c}=3.0$. Relaxation patterns at different $L / R(8.1 ; 8.2 ; 8.5)$ and $\lambda=0.2$. $t^{*}=t \sigma / \eta_{2} R$.

low), the restabilization situation observed here seems to be new.

It could be tempting to increase further the capillary number in order to observe more complex sequences. We tested the case $\lambda=0.2, C a / C a_{c}=3$ presented in Fig.5. We find a larger critical value of 8.15 for $(L / R)_{c}$ in this case, in agreement with the experiments, but the overall fragmentation sequence seems to be simpler than the previous one. We shall however mention that we do not observe the fragmentation into two droplets for this set of parameters, instead, the drop relaxes into three droplets, after cessation of flow, above the critical stretch ratio. For this computation, as the capillary number is rather larger due to the large elongation rate used (steep increase of $L / R$ in time), the time step has been reduced to avoid instabilities, due to the finite difference scheme used.

These three examples illustrate quite well the complexity of the fragmentation sequences, and the non trivial dependence on the control parameters. We could observe a high sensitivity of the results to the control parameters, in particular the restabilization observed in the second example presented above only exists in a very narrow range of parameters (between $L / R=7.9$ and $L / R=8.0$ ) that could be easily missed experimentally.

To complete the comparison with the experimental work of Ha and Leal, we plot the critical stretch ratios obtained for $\lambda=0.2$ in Fig.6.

These values correspond to the first transition from a sphere to two or more spherical droplets plotted as a function of the capillary ratio $C a / C a_{c}$. The capillary numbers are all higher than the critical one, to be able to obtain droplet breakup. Also shown are the experimental data by Ha and Leal (Fig.3 of their paper), that are found to be in qualitative agreement. The discrepancy might be due to the difficulty to control the stretching 


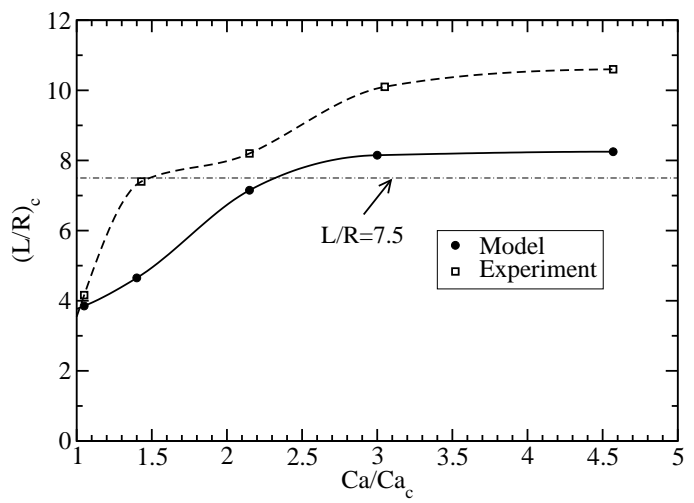

FIG. 6: Critical $L / R$ vs. $C a / C a_{c}$ at a viscosity ratio $\lambda=0.2$.

rate accurately in the experiments. Another explanation may be that, to become more accurate, a smaller value of $\epsilon$ is needed in the simulations, this was not possible due to the large systems we had to consider. We can only mention that in 2D our results compared very well with the analytical calculation by Buckmaster and Flaherty (Fig.2) for similar values of $\epsilon$.

\section{B. Influence of the capillary number}

In the previous part, we observed that the capillary number plays an important but complex role in the restabilization process. Our purpose is now to identify more precisely this role. To this aim, we shall consider a fixed elongation ratio $L / R=7.5$, and investigate the relaxation sequence as a fonction of the capillary number used to produce the elongated shape. We thus follow the horizontal path plotted in Fig.6. This value has been chosen to match the data of Ha and Leal (Figures 2 in Ref. [40]), still at a viscosity ratio of 0.2 . Figs $7 \mathrm{a}-\mathrm{c}$ show the different relaxation mechanisms, following stretching rates corresponding to capillary numbers of $0.212,0.326$ and 0.455 respectively.

The first breaking pattern leads to the formation of a long elongated droplet in the center, which eventually breaks due to end-pinching [15-18], thus forming three droplets. In the second case, larger side droplets are formed and the central resulting droplet is smaller, but they are still three. Finally, as the capillary number (i.e. the elongational rate) is increased, the initial shape of the droplet is different, exhibiting almost pointed ends, as expected for such a small viscosity ratio. This feature is due to the fast elongation process, inhibiting the creation of bulbed ends.

Whereas the elongated drops present bulbed ends at small capillary numbers, favoring fragmentation in a relaxation experiment, they do not present these features
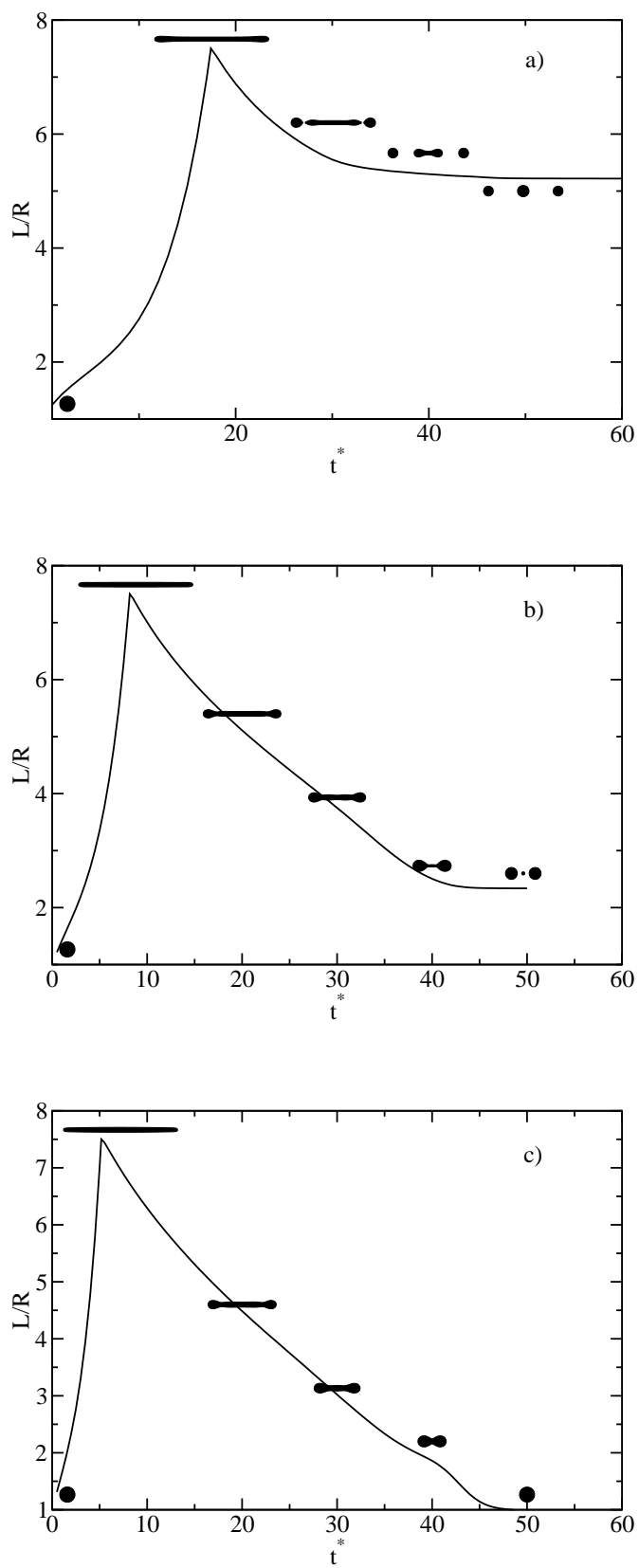

FIG. 7: Relaxation patternfollowing elongation. Stretch ratio $L / R=7.5 . \quad \lambda=0.2 . C a_{c}=0.151$.

$$
\begin{aligned}
& \text { a) } C a=0.212, C a / C a_{c}=1.4 \text { b) } C a=0.326, \\
& C a / C a_{c}=2.15 \text { c) } C a=0.455, C a / C a_{c}=3 .
\end{aligned}
$$

at large capillary numbers. In this case, the bulbed ends responsible for the break-up process must be produced during the relaxation, which can take time. When relaxation is not efficient enough for the drop to develop bulbed ends rapidly as time goes on, the droplet relaxes to a sphere.

Large values of $\mathrm{Ca} / \mathrm{Ca}_{c}$ thus do not favor the fragmen- 
tation, and the most interesting restabilization sequences were observed around $\mathrm{Ca} / C a_{c}=2.11-2.15$, at $\lambda=0.2$.

\section{Restabilization}

In this part we investigate the restabilization sequence in more details. We would like to emphasize that this feature is generic, and can be observed for many values of the parameters. Moreover, we also would like to show that such restabilization sequences are very sensitive to the precise values of the parameters and can present a huge richness. We thus used a set of parameters also considered by Ha and Leal [40] $\left(\lambda=0.056\right.$ and $\mathrm{Ca} / \mathrm{Ca}_{c}=$ $2.15)$, and we shall compare results with the previous restabilization sequence $\left(\lambda=0.2\right.$ and $\left.C a / C a_{c}=2.11\right)$.

The observations are shown in Fig.8, where the droplet has been elongated from its initial spherical shape to a certain degree of elongation characterized by a value of $L / R$, at a capillary number $C a=0.359$. For this case, the critical capillary number is found to be $C a_{c}=0.167$ (thus $\mathrm{Ca} / \mathrm{Ca} a_{c}=2.15$ ). An interesting series of droplet breakups is observed. This scenario is very similar to the experiments of Ha and Leal:

- at first the droplet is elongated but relaxes to a single sphere $L / R=6.9$

- then the droplet breaks up into two spheres $L / R=$ 7.1

- the droplet restabilizes and goes back into a sphere $L / R=7.3$

- the droplet breaks into three droplets $L / R=7.5$

- the central droplets disappears and two droplets are obtained $L / R=7.7$

- the central drop is formed again and we end up with three drops $L / R=8.5$

As expected from the work of Ha and Leal [40], these results confirm the idea that the mechanism of relaxation, which is governed by two parameters, $\lambda$ and the initial shape of the droplet, is based on the following idea: a droplet relaxes by forming first bulbed ends, which need to have enough time to develop [12]. After this deformation is achieved, the droplet motion is governed by the stability of the filament, which can be described accurately by linear stability analysis $[12,20]$. Following the work of Tomotika [20, 21], predictions of the most unstable mode can be obtained when the viscosity ratio $\lambda$ is known. Of course, this study has been carried out when the outer fluid is at rest, but nevertheless it seems to predict rather well the onset of stability [12]. Analysis of the previous droplet shapes reveal that, after bulbed ends are formed, the length of the filament seems to predict rather well the evolution of the droplet to an unstable situation or not.

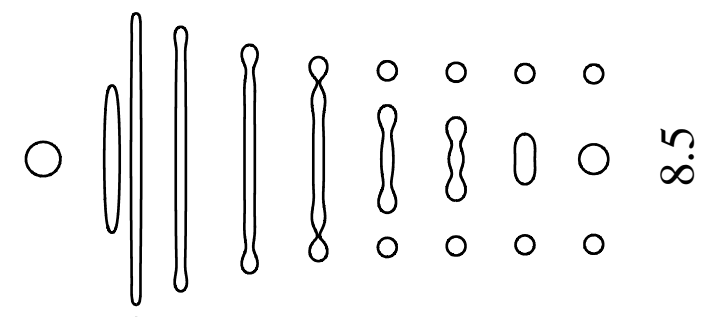

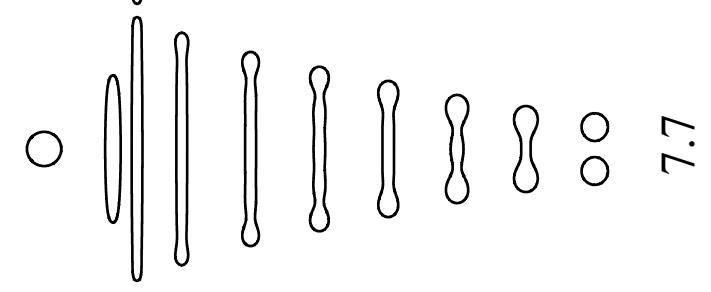<smiles></smiles><smiles></smiles><smiles></smiles><smiles>O=C=O</smiles>

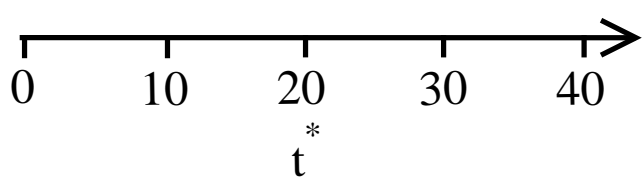

FIG. 8: Relaxation of droplets after stretching at a given $L / R$ $\left(C a / C a_{c}=2.15, \lambda=0.056, C a_{c}=0.167\right)$. 
Another interesting aspect is the restabilization of the droplet independently of its size, as revealed by the succession of shapes in Fig.8. From $L / R=7.1$ to $L / R=7.3$, the droplet restabilizes. From $L / R=7.5$ to $L / R=$ 7.7 , restabilization is also obtained, but now the central droplet has disappeared. This phenomenon is also observed at different viscosity ratios, and could be universal. Even in this case the restabilization differs from the situation presented in Table II by the fact that the central fragments for $\lambda=0.2$ are small satellite droplets whereas for $\lambda=0.056$ they correspond to the largest fragment.

A case of interest would be to follow systematically the fragmentation process as $L / R$ is increased and for a fixed $\lambda$. This should allow to determine whether fragmentation is governed by a deterministic process or exhibits a chaotic behavior. This still requires to improve the numerical scheme presented here in terms of precision (i.e. smaller values of $\epsilon$, mesh adaptation, etc.) in order to capture the formation of smaller daughter droplets including satellites. This work is currently under way.

\section{CONCLUSION}

We presented a comparison between the elongationrelaxation experimental data obtained by $\mathrm{Ha}$ and Leal and the numerical data obtained with the Advected Field method. Although we observe quantitative differences between 2D shear simulations and 3D experimental data, which can be explained by the difference of dimensionality, the numerical data obtained in $3 \mathrm{D}$ is in very good agreement with the experimental findings. In particular, the method is able to reproduce the complex fragmentation-restabilization sequence observed experimentally, and may give the possibility to investigate simpler drop geometries such as the spherocylindrical shape. The observed behaviors are deterministic, and the restabilization sequences are responsible for the apparent noise in the data. However this poses the problem of the influence of thermal fluctuations. These fluctuations have not been introduced in the model, but are expected to become important when the drop is elongated. Indeed, a thermal noise in the problem could reduce the restabilization processes, and reduce the data dispersion. This is of course a conjecture that needs to be further investigated.

\section{Acknowledgements}

The authors are grateful to the "Institut de Physique de la Matière Condensée" (IPMC) for a special grant devoted to computations.
[1] G.I. Taylor, "The viscosity of a fluid containing small drops of another fluid", Proc. R. Soc. A138, 41 (1932).

[2] G.I. Taylor, "The formation of emulsions in definable fields of flow", Proc. Royal Soc. A146, 501 (1934).

[3] H.P. Grace, "Dispersion phenomena in high viscosity immiscible fluid systems and application of static mixers as dispersion devices in such systems", Chem. Eng. Commun. 14, 225 (1982).

[4] B.J. Bentley, L.G. Leal, "An experimental investigation of drop deformation and breakup in steady twodimensional linear flows", J. Fluid Mech. 167, 241 (1986).

[5] S. Torza, R.G. Cox, S.G. Mason, "Particle motions in sheared suspensions", J. Colloid Int. Sci. 38, 395 (1972).

[6] D. Barthès-Biesel, A. Acrivos, "Deformation and burst of a liquid droplet freely suspended in a linear shear field", J. Fluid. Mech. 61(1), 1 (1973).

[7] J.D. Buckmaster, "Pointed bubbles in slow viscous flow", J. Fluid Mech. 55(3), 385 (1972)

[8] E.J. Hinch, A. Acrivos, "Steady long slender droplets in two-dimensional straining motion", J. Fluid Mech. 91(3), 401 (1979).

[9] A. Acrivos, T.S. Lo, "Deformation and breakup of a single slender drop in an extensional flow", J. Fluid Mech. 86(4), 641 (1978).

[10] O.A. Ladyzhenskaya, "The mathematical theory of viscous incompressible flow", 2nd edition, Gordon \& Breach (1969).

[11] J.M. Rallison, A. Acrivos, "A numerical study of the deformation and burst of a viscous drop in an extensional flow", J. Fluid. Mech. 89(1), 191 (1978).
[12] H.A. Stone, L.G. Leal, "Relaxation and breakup of an initially extended drop in an otherwise quiescent fluid", J. Fluid Mech. 198, 399 (1989).

[13] A.Z. Zinchenko, M.A. Rother, R.H. Davis, "A novel boundary-integral algorithm for viscous interaction of deformable drops", Phys. Fluids, 9(6), 1493 (1997).

[14] C. Pozrikidis, "Boundary integral and singularity methods for linearized viscous flows", Cambridge University Press, Cambridge (1992)

[15] J. Eggers, "Nonlinear dynamics and breakup of freesurface flows", Rev. Modern Phys. 69(3), 865 (1997).

[16] J.R. Lister, H.A. Stone, "Capillary breakup of a viscous thread surrounded by another viscous fluid", Phys. Fluids 10(11), 2758 (1998).

[17] I. Cohen, M.P. Brenner, J. Eggers, S.R. Nagel, "Two fluid drop snap-off problem: experiments and theory", Phys. Rev. Letters 83(6), 1147 (1999).

[18] D.R. Webster, E.K. Longmire, "Jet pinch-off and drop deformation in immiscible liquid-liquid systems", Exp. Fluids 30, 47 (2001).

[19] Y.Y. Renardy, V. Cristini, "Scalings for fragments produced from drop breakup in shear flow with inertia", Phys. Fluids 13(8), 2161 (2001).

[20] S. Tomotika, "On the instability of a cylindrical thread of a viscous liquid surrounded by another viscous fluid", Proc. R. Soc. London A150, 322 (1935).

[21] S. Tomotika, "Breaking up of a drop of viscous liquid immersed in another viscous fluid which is extending at a uniform rate", Proc. Royal Soc. London A153, 302 (1936). 
[22] M. Sussman, P. Smereka, S. Osher S, "A level set approach for computing solutions to incompressible twophase flow", J. Comput. Physics 114, 146 (1994).

[23] M. Sussman, E. Fatemi, P. Smereka, S. Osher, "An improved level set method for incompressible two-phase flows", Computers and Fluids 27(5-6), 663 (1998).

[24] S.B. Pillapakkam, P. Singh, "A Level Set Method for computing solutions to viscoelastic two-phase flow", J. Comput. Physics 174, 552 (2001).

[25] J. Li, Y.Y. Renardy, M. Renardy, "Numerical simulation of breakup of a viscous drop in simple shear flow through a volume-of-fluid method", Phys. Fluids 12(2), 269 (2000).

[26] J. Beaucourt, F. Rioual, T. Sèon, T. Biben, C. Misbah, "Steady to unsteady dynamics of a vesicle in a flow", Phys. Rev. E 69, 011906 (2004).

[27] T. Biben, C. Misbah, A. Leyrat, C. Verdier, "An advected-field approach to the dynamics of fluid interfaces", Europhys. Letters 63(4), 623 (2003).

[28] P. Yue, J.J. Feng, C. Liu, J. Shen, " A diffuse-interface method for simulating two-phase flows of complex fluids ", J. Fluid Mech. 515 , 293 (2004).

[29] H. Haj-Hariri, Q. Shi, A. Borhan, " Effect of local property smearing on global variables : implication for numerical simulations of multiphase flows", Phys. Fluids, 6(8), 2555 (1994).

[30] R. Chella, J. Vinals, " Mixing of a two-phase fluid by cavity flow", Phys. Rev. E, 53(4), 3832 (1996).

[31] D.M. Anderson, G.B. McFadden, A.A. Wheeler, " A phase-field model of solidification with convection ", Physica D, 135, 175 (2000).

[32] J. Li, Y.Y. Renardy, "Shear-induced rupturing of a viscous drop in a Bingham liquid", J. Non-Newtonian Fluid Mech. 95, 235 (2000).

[33] Y.T. Hu, D.J. Pine, L.G. Leal, "Drop deformation, breakup, and coalescence with compatibilizer", Phys. Fluids 12(3), 484 (2000).

[34] H.A. Stone, L.G. Leal, "The effects of surfactants on drop deformation and breakup", J. Fluid Mech. 220, 161 (1990)

[35] D. Barthès-Biesel, H. Sgaier, "Role of membrane viscosity in the orientation and deformation of a spherical capsule suspended in shear flow", J. Fluid Mech. 160, 119 (1985).

[36] G. K. Batchelor, "The stress system in a suspension of force-free particles", J. Fluid Mech. 41, 545 (1970).

[37] N.A. Frankel, A. Acrivos, "The constitutive equation for a dilute emulsion", J. Fluid Mech. 44(1), 65 (1970).

[38] K.M.B. Janssen, W.G.M. Agterof, J. Mellema, "Droplet breakup in concentrated suspensions", J. Rheol., 45(1), 227 (2001).

[39] D.C. Tretheway, L.G. Leal, "Deformation and relaxation of Newtonian drops in planar extensional flows of a Boger fluid", J. Non-Newtonian Fluid Mech. 99, 81 (2001).

[40] J.-W. Ha, L.G. Leal, "An experimental study of drop deformation and breakup in extensional flow at high capillary number", Phys. Fluids, 13(6), 1568 (2001).

[41] J.S. Rowlinson, B. Widom," Molecular Theory of Capillarity" (Oxford University Press, Oxford, 1989).

[42] J.W. Cahn, S.M. Allen, " Microscopic theory for domain wall motion and its experimental verification in $\mathrm{Fe}-\mathrm{Al}$ alloy domain growth kinetics", J. Phys. (Paris) Colloq. Suppl. C7, 51 (1977).

[43] S.M. Allen, J.W. Cahn, " A microscopic theory for antiphase boundary motion and its application to antiphase domain coarsening ", Acta Mettal. 27, 1085 (1979).

[44] D. Jasnow, J. Vinals, "Coarse-grained description of thermo-capillary flow", Phys. Fluids 8(3), 660 (1996).

[45] R. Folch, J. Casademunt, A. Hernandez-Machado, L. Ramirez-Piscina, "Phase-field model for Hele-Shaw flows with arbitrary viscosity contrast. I. Theoretical approach", Phys. Rev. E 60(2), 1724 (1999).

[46] R. Almgren, " Variational algorithms and pattern formation in dendritic solidification ", J. Comput. Phys. 106, 337 (1993).

[47] A.A. Wheeler, B.T. Murray, R.J. Schaefer, " Computation of dendrites using a phase filed model, Physica D 66, 243 (1993).

[48] J.D. Buckmaster, J.E. Flaherty, "The bursting of twodimensional drops in slow viscous flow", J. Fluid Mech. 60(4), 625 (1973).

[49] H.A. Stone, B.J. Bentley, L.G. Leal, "An experimental study of transient effects in the breakup of viscous drops", J. Fluid Mech. 173, 131 (1986).

[50] H.A. Stone, "Dynamics of drop deformation and breakup in viscous fluids", Ann. Rev. Fluid Mech. 26, 65 (1994).

[51] H.A. Stone, L.G. Leal, "The influence of initial deformation on drop breakup in subcritical time-dependent flows at low Reynols numbers", J. Fluid Mech. 206, 223 (1989). 